\title{
Analysis and measures of commutation failure in HVDC transmission Erjia Liu
}

\author{
North China Electric Power University, Beijing 102200, China \\ liuerjia62jia@163.com
}

\begin{abstract}
The commutation failure in HVDC inverter is one of the most common faults. Based on the inverter commutation process, this paper analyzes the work of the inverter commutation process and the reason for commutation failure. Learning from the commutation current time area method, it puts forward the method of minimum turn-off area, and compares the difference between it and minimum extinction angle criterion. Control parameters, operating conditions, the state of DC side will all have an impact on the turn-off area. The application of the CIGRE standard model in PSCAD/EMTDC briefly compared and proved the two methods , proving the correctness of the minimum turn-off area method.
\end{abstract}

Key words: commutation failure criterion; extinction angle; commutation; turn off area

\section{Introduction}

In long distance, large power grid interconnection and capacity transmission HVDC is applied widely. As the the most common typical fault in HVDC system, commutation failure will cause DC voltage drop and DC current short-term elevation. when the fault is serious or even causes the DC power transfer completely to be interrupted, this is very unfavorable to the DC transmission. So we should take corresponding measures to solve them. This paper starts with the commutation process followed by a detailed study of the two valve commutation process, proposing the method to analyse the two valve dynamic process and the relationship between the extinction angle by the virtue of minimum shutdown area, revealing the phase change mechanism in essence.

\section{Commutation failure mechanism}

\subsection{Definition of commutation failure}

valve arm of an inverter in a power cycle is under positive voltage drop most of the time. The definition of commutation failure: in a inverter, the valve could not complete the commutation process or restore the blocking capacity in the period of reverse voltage after exiting the conduction, whose anode voltage will then be greater than zero and re-conduct out of control.When Commutation failure occurs, the two valves within one circulation will turn on at the same time. DC current will increase rapidly at the same conduction time. It will not only make the transmission voltage and current transfer DC side zero, it is more serious that short current will increase the commutation overlap angle causing the commutation of the following valve arm of the inverter to fail, which will finally lead to the duration of the DC short-circuit fault, resulting in the action of protection system and the power failure of the whole transmission line. 


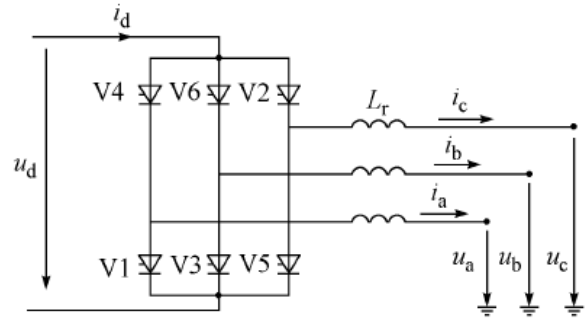

Fig 1

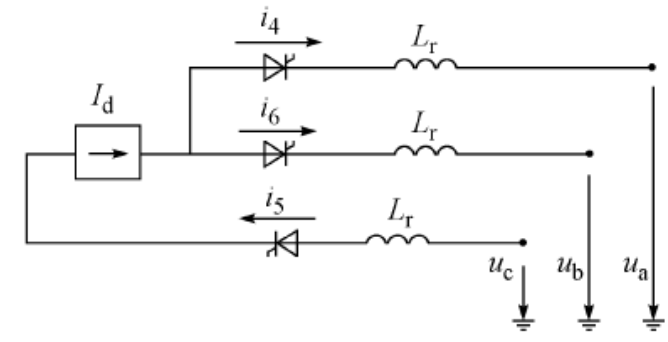

Fig 2

\subsection{Commutation mechanism}

Take the valve V4 commutating to valve V6 for example: before V6 opened, V4 and V5 conduct. After V6 conducts, V4 V5, V6 conduct at the same time. Because of the commutation inductance Lr, commutation current does not chage abruptly. During the commutation time, current through valve V4 will drop from DC current Id to zero,meanwhile, current through valve V6 will rise from zero to DC current Id. However, when current through V4 valve decrease to 0, the valve has not turned off yet, since after V4 quits conduction, the carrier recombination of in the thyristor will take some time to build the P-N junction barrier to restore its ability to block forward voltage. The deionization recovery time is usually about $400 \mu \mathrm{s}$ (around $7^{\circ}$ electric angle), $\gamma_{\min }$ is often set $8^{\circ}$. When extinction angle is less than $15^{\circ} \sim 18^{\circ}$, commutation failure is deemed to occur, taking account of the margin for asymmetric parameters of three-phase voltage in AC system. [1]

Theoretical calculation of commutation process

Ignoring the forward voltage drop of the thyristor, two voltage loop equations can be listed by Kirchhoff's law:

$$
\begin{aligned}
& L_{r} \frac{d I_{d}}{d t}+u_{c}-u_{a}+L_{r} \frac{d i_{v_{4}}}{d t}=U_{d} \\
& L_{r} \frac{d I_{d}}{d t}+u_{c}-u_{b}+L_{r} \frac{d i_{v_{6}}}{d t}=U_{d}
\end{aligned}
$$

Take the time when $u_{c b}$ cross zero point as initial time, $\mathrm{U}$ as effective value of AC side voltage:

$$
u_{C B}=\sqrt{2} U \sin \left(\omega t+\frac{2 \pi}{3}\right)
$$

According to (1) (2) (3):

$$
\gamma=\arccos \left(\frac{\sqrt{2} I_{d} X_{r}}{U}+\cos \beta\right) \quad, X_{r}=\omega L_{r}
$$

(4) can be obtained from the turn-off angle $\gamma$, DC current ${ }^{I_{d}}$, AC side line voltage value $\mathrm{U}$, the trigger angle $\beta$ and commutation reactance of $\mathrm{Xr}$. So the common discrimination method of commutation failure is the minimum turn-off angle criterion: when the trigger angle is less than the minimum turn-off angle the commutation failure will occur. 
There are still some defects in the method of minimum turn off angle,which assumes that the DC current and the trigger angle is constant, but in the actual fault condition, voltage waveform distortion will have an influence on turn-off angle discrimination, may causing unavoidable error. So the project adopting minimum turn-off area method is more reliable.

The expressions of commutation time-area can also be derived from (1) (2) (3):

$$
A=\int_{\pi-\beta}^{\pi-\gamma} \sqrt{2} U \sin (\omega t) d(\omega t)=2 X_{r} I_{d}
$$

Tut-off area:

$$
G=\int_{\pi-\gamma}^{\pi} \sqrt{2} U \sin (\omega t) d(w t)=\sqrt{2} U(1-\cos \gamma)
$$

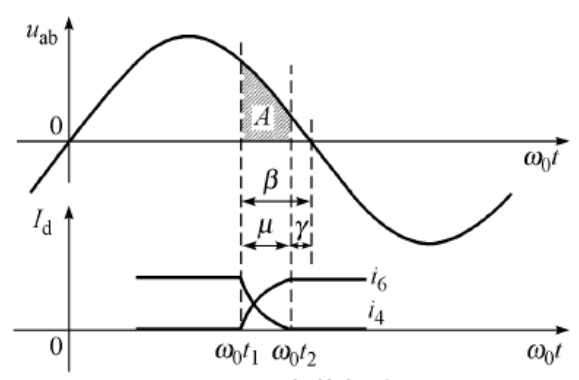

Fig 3

Choosing the minimum turn-off area has considered not only the influence of turn-off angle, but also the influence of AC voltage. In the normal operation of HVDC system, the AC voltage will maintain a constant value. If the external fault occurs, the AC voltage drop, DC current rise instantaneously, and the commutation inductance will accumulate a large amount of energy, so it takes more time to release the energy accumulated. If the turn-off area is too small, commutation failure may occur . As $\beta$ increases, turn-off area will increase, commutation failure is more likely to avoid with more margin for error.So we can set a minimum turn-off area:

When turn-off area is under the minimum, commutation failure occurs. It is because the turn-off angle is too small or thyristor has not endured enough reverse voltage to recover the recombination of charge carrier.However, it should be noticed that the minimum turn-off area cannot be infinitely small. We need sample the minimum amplitude of the instantaneous three-phase AC voltage in the inverter, then based on the formula (4), we can set a reference value with a upper and a lower limit, from the reference, the maximum of trigger angle can be calculated.

$$
\gamma_{\text {ref }}=\arccos \left(1-\frac{1-\cos \gamma_{\min 0}}{U / U_{N}}\right)
$$

Obviously, when turn-off area is larger than the reference, the commutation angle $\mu$ is less than the given commutation angle maximum, meanwhile, the extinction angle $\gamma$ is greater than the given minimum extinction angle, failure is not likely to occur; when turn-off area is less than the reference, both $\mu$ and $\gamma$ will be beyond the limit and failure occurs, therefore, measures should be taken.

We usually take measures to control commutation failure or manage to recover after failuer. There are many measures: taking reactive compensation to stabilize the phase voltage, adopting larger smoothing reactor, reducing the short circuit impedance of converter transformer, taking appropriate control mode, forced commutation and series of restoration strategy. To not specify, please refer to literature [2].

This essay is mainly discussing the influence of turn-off area, there are still some essays to research the the time area of commutation voltage. Two have some similarities.

3 simulated analysis

In order to verify the accuracy of minimum turn-off current, we take CIGER standard model of PSCAD/EMTDC 
simulation software to compare theoretical and actual value.Standard parameters of GIGRE inverter side: DC current 1.0p.u. (2kA) ,Converter bus voltage 1.0p.u.(230kA), Converter voltage ratio 1.0p.u.(230/209.23), Single-phase commutation reactance $18 \%$ (p.u.). According to the extinction angle model in [5] we can duduce the extinction angle, and the relationship between actual extinction angle and DC current.

It can be seen that the turn-off angle has a negative correlation with the DC current. The larger the DC current is, the smaller the extinction angle of the conventional inverter is. Not enough time to withstand reverse voltage will lead to commutation failure, which coincides with the previous deduction.

When $\beta$ is $50^{\circ}$, the calculated, simulated value of turn-off angle and area Tab 1

\begin{tabular}{ccccc}
\hline $\begin{array}{c}\text { Direct current } \\
\text { (p. u. })\end{array}$ & calculated value $\gamma$ & $\begin{array}{c}\text { Simulated } \\
\text { value } \gamma\end{array}$ & turn-off area & Minimum turn-off area \\
\hline 1.0526 & 13.125 & 14.5 & 0.045 & 0.0138 \\
1.1111 & 10.9345 & 12.31 & 0.0325 & 0.0138 \\
1.1765 & 8.2664 & 9.98 & 0.0213 & 0.0138 \\
1.2500 & 4.8885 & 6.34 & 0.00865 & 0.0138 \\
1.3333 & 0.2911 & 0 & 0 & 0.0138
\end{tabular}

As can be seen from the table, only when $\beta$ is $50^{\circ}$ can minimum turn-off area criterion distinguish Commutation failure effectively, which coincides with extinction angle criterion, so minimum turn-off area is advisable. In addition, with the disturbance of AC voltage, minimum turn-off area control strategy performs much better with a high accuracy.

However, there are still some problems with this method: On the one hand, when the AC voltage drops greatly, the minimum shutoff area cannot be used, On the other hand, the extinction angle is the difference between $\beta$ and $\mu$, So commutation failure and commutation process are also related, However, this method does not consider the commutation process enough.

\section{Conclusion}

This paper starts with the mechanism of commutation, the difference and relation between the two commutation failure criterion are compared.The relationship between commutation voltage, area, turn-off area, DC current and AC voltage has been discussed.Operating parameters(extinction angle, DC current etc.) of the system can be utilized to predict and react when the fault occurs in the shortest time. We should consider the external factors to choose the criterion. It is better to adopt the minimum turn-off area method when the AC voltage is disturbed greatly.

\section{Reference}

[1] Han Minxiao, Wen Jun, Xu Yonghai. Principle and operation of HVDC transmission

[2]Yuan Yang, Wei Zhinong, Wang Huawei, Lei Xiao, Sun Guoqiang. Review of HVDC systems commutation failure [J]. automation of electric power equipment, 2013, (11): 140-147.

[3] Li Sisi, Bai Li, Ding Zhilin, Wang Yuhong, Li Xingyuan, Wei Wei, He Pengfei. Commutation failure detection method based on time domain of commutation current [J]. Journal of power system and automation,2013,(02):98-102.

[4]Wang Gang, Li Zhikeng, Huang Min, Li Haifeng. HVDC. Failure mechanism of switching failure in transmission system [J]. automation of electric power systems, 2010, (04): 49-54+102.

[5] Ou Kai Jian, Ren Zhen, Jing Yong, et al. Study on commutation failure in HVDC transmission system (a) analysis: commutation failure factors. Electric power automation equipment, 2003,23 (5):5-8,25. 